Kaliano Márcio de Queiroz Costa'

(D) https://orcid org/0000-0003-2203-401X

Rachel Medeiros de Góes²

Onttps://orcid.org/0000-0002-6566-2369

Maria Mabel Nunes de Morais

O- https://orcid.org/0000-0001-6738-7316

\section{A influência dos aspectos subjetivos na adesão ao tratamento do transtorno bipolar: uma revisão sistemática}

\author{
The influence of subjective aspects on adherence to the \\ treatment of bipolar disorder: a systematic review
}

DOI: $10.1590 / 0047-2085000000355$

\begin{abstract}
RESUMO
Objetivo: Evidenciar a influência dos aspectos subjetivos na adesão ao tratamento do transtorno bipolar. Métodos: Foi realizada revisão sistemática com base nas diretrizes PRISMA. A identificação dos estudos foi realizada por meio da busca nos bancos de dados PubMed, Scopus e SciELO, com base nos descritores "Bipolar Disorder" AND "Treatment Adherence and Compliance" AND "Mental Health". A busca contemplou todos os artigos publicados até o ano 2020, sem restrição de idioma. Resultados: Foram localizados 743 artigos, 714 foram excluídos no processo de seleção, 29 foram lidos na íntegra e 11 foram elegíveis para a composição da amostra. A influência dos aspectos subjetivos na adesão ao tratamento foi associada (1) às atitudes resultantes das percepções do sujeito sobre o transtorno e o tratamento e (2) as atitudes por influência de pessoas próximas. Os estudos apontam para a ocorrência de atitudes negativas em ambas as esferas, tendo a má adesão ao tratamento como desfecho. Na esfera da percepção do sujeito, evidenciam-se: presença de comportamentos intencionais e não intencionais; percepção de consequências; medo dos efeitos colaterais; sentimentos negativos; falta de compreensão sobre o transtorno e negação do diagnóstico. Na esfera da influência das pessoas próximas, destacam-se a baixa qualidade da aliança terapêutica e o suporte ineficaz oferecido pela família. Conclusões: Para melhorar a adesão ao tratamento do transtorno bipolar, é salutar que os esforços terapêuticos estejam centrados na experiência particular do sujeito, na sua satisfação e na colaboração pactuada com o tratamento.
\end{abstract}

PALAVRAS-CHAVE

Transtorno bipolar, cooperação e adesão ao tratamento, saúde mental.

\begin{abstract}
Objective: Evidence the influence of subjective aspects on adherence to the treatment of bipolar disorder. Methods: A systematic review was performed based on the PRISMA guidelines. The identification of studies was performed by searching the PubMed, Scopus and Scielo databases based on the descriptors "Bipolar Disorder" AND "Treatment Adherence and Compliance" AND "Mental Health". The selection included all articles published up to the year 2020 and without language restrictions. Results: A total of 743 articles were found, 714 were excluded from the selection process, 29 articles were read in full and 11 were eligible for sample composition. The influence of subjective aspects on treatment adherence was associated (1) with attitudes resulting from the subject's perceptions about the disorder and treatment and (2) attitudes influenced by people close to them. Studies point to the occurrence of negative attitudes in both spheres, with poor adherence to treatment as an outcome. In the sphere of the subject's perception, they show the presence of intentional and unintentional behaviors; perception of consequences; fear of side effects; negative feelings; lack of understanding about the disorder and denial of diagnosis. In the sphere of influence of those close to them, they highlight the low quality of the therapeutic alliance and the ineffective support offered by the family. Conclusions: To improve adherence to treatment for bipolar disorder, it is beneficial that therapeutic efforts are centered on the individual's particular experience, on their satisfaction and on the agreed collaboration with the treatment.
\end{abstract}

\section{KEYWORD}

Bipolar disorder, cooperation and treatment adherence, mental health.

Received in: July/8/2021. Approved in: Sep/14/2021

1 Universidade Federal do Rio Grande do Norte, Programa de Residência Integrada Multiprofissional em Saúde, Natal, RN, Brasil.

2 Hospital Universitário Onofre Lopes, Natal, RN, Brasil.

3 Universidade Federal do Rio Grande do Norte, Programa de Pós-graduação em Psicologia, Natal, RN, Brasil.

Address for correspondence: Kaliano Márcio de Queiroz Costa. Av. Nilo Peçanha, 620, Petrópolis - 59012-300 - Natal, RN, Brasil. Telefone: (84) $3342-5236$. E-mail: kaliano.mqc@gmail.com 


\section{INTRODUÇÃO}

Segundo dados do projeto colaborativo World Mental Health Survey Initiative da Organização Mundial da Saúde, que conduziu estudos em 11 países nas Américas, Europa e Ásia, o transtorno bipolar (TB) tem taxa de prevalência durante a vida de $0,6 \%$ no tipo I, $0,4 \%$ no tipo $I, 1,4 \%$ no TB subliminar e 2,4\% para todo o espectro bipolar'. Um estudo de seguimento de 4,5 anos mostrou que somente $41 \%$ das pessoas com TB do tipo I tiveram desfecho clínico satisfatório, 37\% apresentaram prejuízo moderado e $22 \%$ tiveram funcionamento pobre 2 . Os impactos para os sujeitos em questão vão desde a recorrência das crises até taxas elevadas de suicídio. Como na maioria dos transtornos mentais, não só o sujeito está adoecido, junto com ele toda a rede social composta por familiares e amigos também sofre as consequências de um tratamento ineficaz ${ }^{3}$.

No que tange ao tratamento farmacológico, o carbonato de lítio, o ácido valproico (estabilizadores de humor), a quetiapina e a olanzapina (antipsicóticos atípicos) são tidos atualmente como opções farmacológicas de primeira linha ${ }^{4}$. Embora os fármacos apresentem eficácia significativa no controle da sintomatologia do transtorno, a maioria dos pacientes tende a conviver com vários efeitos colaterais: tontura, aumento de peso, boca seca, letargia, sedação e déficits cognitivos. O lítio ainda apresenta risco de intoxicação se não for monitorada a sua concentração no organismo 5 . A descoberta do efeito do carbonato de lítio na regulação do humor tornou-o por muitos anos a única opção empregada no tratamento do TB. Mas pesquisas desenvolvidas nos Estados Unidos pelo National Institutes of Health em 1988 evidenciaram elevadas taxas de recaída em pacientes que faziam uso apenas de fármacos, impulsionando pesquisas que destacam os benefícios das intervenções psicossociais no tratamento ${ }^{6}$. No campo dos tratamentos não farmacológicos, as intervenções psicoterapêuticas que obtiveram resultados melhores em estudos foram: terapia cognitivo-comportamental, terapia focada na família e estratégias psicoeducativas. Tem-se o entendimento de que elas atuam de modo adjuvante ao tratamento farmacológico, tendo como principais benefícios uma melhor compreensão do transtorno e do tratamento, aumento da resiliência do paciente para situações de estresse, construção conjunta de estratégias de enfrentamento, melhora do processamento reflexivo, meIhor identificação das oscilações no humor e alterações no comportamento, que são aspectos fundamentais para o fortalecimento da adesão ao tratamento, como também tem impacto direto na qualidade de vida?

O conceito de adesão terapêutica pode variar muito entre os autores que estudam a temática, porém existe o consenso de que ela é compreendida como o uso de $80 \%$ ou mais do total de medicamentos prescritos ou outros procedimentos, tendo a observância das doses, horários e tempo de tratamento 8 . A adesão ao tratamento no transtorno mental vem sendo objeto de estudo há aproximadamente 40 anos. Lingam e $\operatorname{Scott}^{9}$ realizaram uma revisão abrangente da literatura e encontraram que de $20 \%$ a $66 \%$ dos pacientes, com mediana de $41 \%$, não aderem ao tratamento com farmacoterapia profilática a longo prazo. Pensar na adesão terapêutica consiste em um desafio que perpassa uma gama multivariada de fatores que englobam a compreensão apurada do transtorno, sua evolução, arsenal terapêutico e aspectos voltados para as atitudes dos pacientes na experiência do adoecimento e tratamento.

É necessário pontuar que a maioria dos estudos sobre adesão no TB foca em fatores que são externos aos pacientes, como nosologia do transtorno, esquemas terapêuticos, acesso aos serviços de saúde, e fatores internos aos pacientes relacionados às atitudes em relação ao tratamento e ao transtorno. Com relação aos fatores externos, pontua-se que o episódio maníaco e a gravidade do episódio depressivo mostraram-se associados à má adesão ${ }^{10}$. A dependência de substâncias psicoativas também esteve associada a má adesão ao tratamento, inclusive o transtorno por uso de substâncias e o transtorno de ansiedade são as principais comorbidades psiquiátricas no TB ${ }^{11}$. Além disso, apenas 25\% dos pacientes com TB de países subdesenvolvidos referem uso de serviços de saúde mental'. E dados do National Comorbidity Survey mostram que somente 9\% dos pacientes que foram acompanhados por clínicos gerais tiveram um tratamento adequado, mas, quando o acompanhamento é realizado por especialistas, esse número sobe para 45\%. Esse é um dado alarmante, pois os clínicos gerais são responsáveis pelo acompanhamento da maioria dos casos e, segundo o exposto, estão fazendo um manejo ineficaz ${ }^{12}$. Com relação aos fatores internos ou aspectos subjetivos, é elencada uma gama variada de atitudes: medo de eventos adversos; estigmas; falta de motivação; aceitação ou negação do transtorno; influência familiar; confiança nos médicos; compreensão da necessidade e do benefício do tratamento ${ }^{8}$.

Feito esse panorama, os dados da literatura mostram um cenário desafiador; existem muitos estudos no campo dos aspectos objetivos, mas poucos no campo dos aspectos subjetivos. Este estudo, então, busca suprir a seguinte lacuna: quais são os aspectos subjetivos envolvidos e como eles impactam a adesão ao tratamento do TB? Trata-se de um esforço para compreender o que já se conhece a respeito dessa temática, o modo como ela é abordada nos estudos, mas também para reforçar a necessidade de que mais estudos sejam produzidos. Dito isso, o objetivo deste trabalho é compreender a influência dos aspectos subjetivos envolvidos na adesão ao tratamento do TB. Aprofundar-se nesse recorte temático é fundamental para a formulação de planejamentos terapêuticos que considerem a satisfação do usuário com o tratamento acordado e que tenham como 
objetivo incentivar o papel ativo do usuário e sua família no processo de cuidado.

\section{MÉTODOS}

Esta revisão sistemática tomou como base as diretrizes PRISMA $^{13}$. Conforme ilustrado na figura 1, a busca foi realizada nos bancos de dados PubMed, Scopus e SciELO, usando os descritores "Bipolar Disorder", "Treatment Adherence and Compliance" e "Mental Health" combinados com o booleano AND, e no SciELO a busca foi realizada usando os descritores "Transtorno Bipolar", "Adesão ao tratamento" e "Saúde Mental" combinados com o booleano AND; os descritores foram escolhidos por meio de consulta ao DeCS da plataforma BVS da BIREME para escolha dos termos mais adequados (fase de identificação). A busca contemplou todos os artigos publicados até o dia 31 de dezembro de 2020 e não foi utilizada restrição de idioma. As etapas de identificação, seleção e elegibilidade, conforme consta na figura 1, foram realizadas por dois pesquisadores independentes (KM e MM). As discordâncias foram debatidas entre os dois pesquisadores até eles chegarem a um consenso. Nos casos que não houve consenso, um terceiro revisor foi consultado para resolver o impasse.

Foram considerados como critérios de inclusão: 1) artigos publicados até o dia 31 de dezembro de 2020; 2) estudos empíricos; 3) estudos voltados especificamente a sujeitos com TB e 4) utilização de metodologias qualitativas e/ou quantitativas para avaliar os aspectos subjetivos (percepção, atitude, motivação, sentimentos etc.). Foram considerados como critérios de exclusão: 1) os trabalhos que tratavam de pessoas com transtornos mentais de modo geral, sem deixar claro quais transtornos estavam sendo estudados; 2) trabalhos que estudavam o TB junto com outros transtornos, como estudos com TB e esquizofrenia e estudos sobre transtornos de humor. Tais critérios foram considerados porque este estudo busca descobrir os aspectos subjetivos específicos das pessoas com TB para que sirva de base para modelos terapêuticos que considerem as peculiaridades inerentes ao transtorno. Também foram excluídos: 3) os artigos que tratavam da adesão atrelada apenas a um psicofármaco, esquema medicamentoso ou método psicoterapêutico específico como forma de comprovar sua eficácia, sem realizar aprofundamento dos fatores que levam à adesão ao tratamento como um todo; 4) os estudos que apenas mediam a adesão ao tratamento sem medir nenhum aspecto subjetivo; 5) estudos que não utilizaram uma metodologia consistente para avaliar a adesão terapêutica e 6) revisões de literatura.

Na identificação, como mostra a figura 1, foi encontrado um total de 743 artigos. Foram identificadas e excluídas 181 duplicatas. Na triagem, foi realizada seleção dos artigos pelo título com base nos critérios de exclusão, (os critérios de exclusão 1 e 2 foram observados com mais frequência nessa fase), restando 124 artigos que tinham alguma relação com a adesão ao TB. Esses 124 artigos tiveram seus resumos avaliados novamente com base nos critérios de exclusão (os critérios de exclusão 3, 4, 5 e 6 foram mais frequentes nessa fase), restando 29 artigos. Na elegibilidade, 29 artigos tiveram seus textos completos analisados e 18 artigos foram excluídos com base nos critérios de exclusão 3, 4 e 5. A exclusão desses artigos nessa fase ocorreu porque na leitura dos resumos não havia ficado claro se eles poderiam ser excluídos; somente após a leitura integral dos artigos é que foi observada a aplicabilidade dos critérios de exclusão. Restaram para a fase de inclusão 11 artigos, que passaram por avaliação de qualidade metodológica com base nos critérios do Mixed Methods Appraisal Too/14. Todos os estudos responderam satisfatoriamente aos quesitos elencados e apresentaram metodologia adequada.

Como o objeto de estudo dessa pesquisa envolve aspectos subjetivos (percepções, atitudes, motivações, sentimentos etc.), que são construtos de difícil mensuração quantitativa, foram aceitos estudos com metodologia qualitativa e/ou quantitativa. Em face disso, a análise foi baseada na metassíntese ${ }^{15}$, conforme os seguintes passos: ambos os pesquisadores realizaram a leitura completa e releituras de todos os artigos incluídos de modo aprofundado, buscando compreender de modo amplo as principais conclusões. Foram elaboradas listas com os temas comuns, diferenças entre os estudos e classificações por áreas e relações. A partir dessas listas, os achados foram agrupados nos temas: 1) atitudes dos sujeitos em relação ao tratamento; 2) percepção do sujeito sobre o tratamento; 3) percepção do sujeito sobre o transtorno; 4) sentimentos do sujeito em relação ao transtorno ou tratamento e 5) influência de pessoas próximas no tratamento. Estabelecidos os temas, foi realizada nova busca nos artigos para verificar contradições ou convergências dos resultados dos estudos incluídos. Por fim, foi criado um fluxograma e síntese dos principais achados.

\section{RESULTADOS}

Estudos envolvendo os aspectos subjetivos que estão associados na adesão ao tratamento do TB são recentes e fazem um contraponto aos estudos tradicionais que se centram em aspectos nosológicos do TB, esquemas terapêuticos e acesso a serviços de saúde. Existem poucos estudos centrados nos aspectos subjetivos e essa é uma temática que ainda necessita de mais pesquisas e melhor uniformidade das estratégias metodológicas para futuras comparações entre realidades diversas. Todos os trabalhos incluídos nesta seção, que estão descritos na tabela 1, apontam como resultados que atitudes negativas em relação ao tratamento são fatores determinantes na adesão terapêutica. 


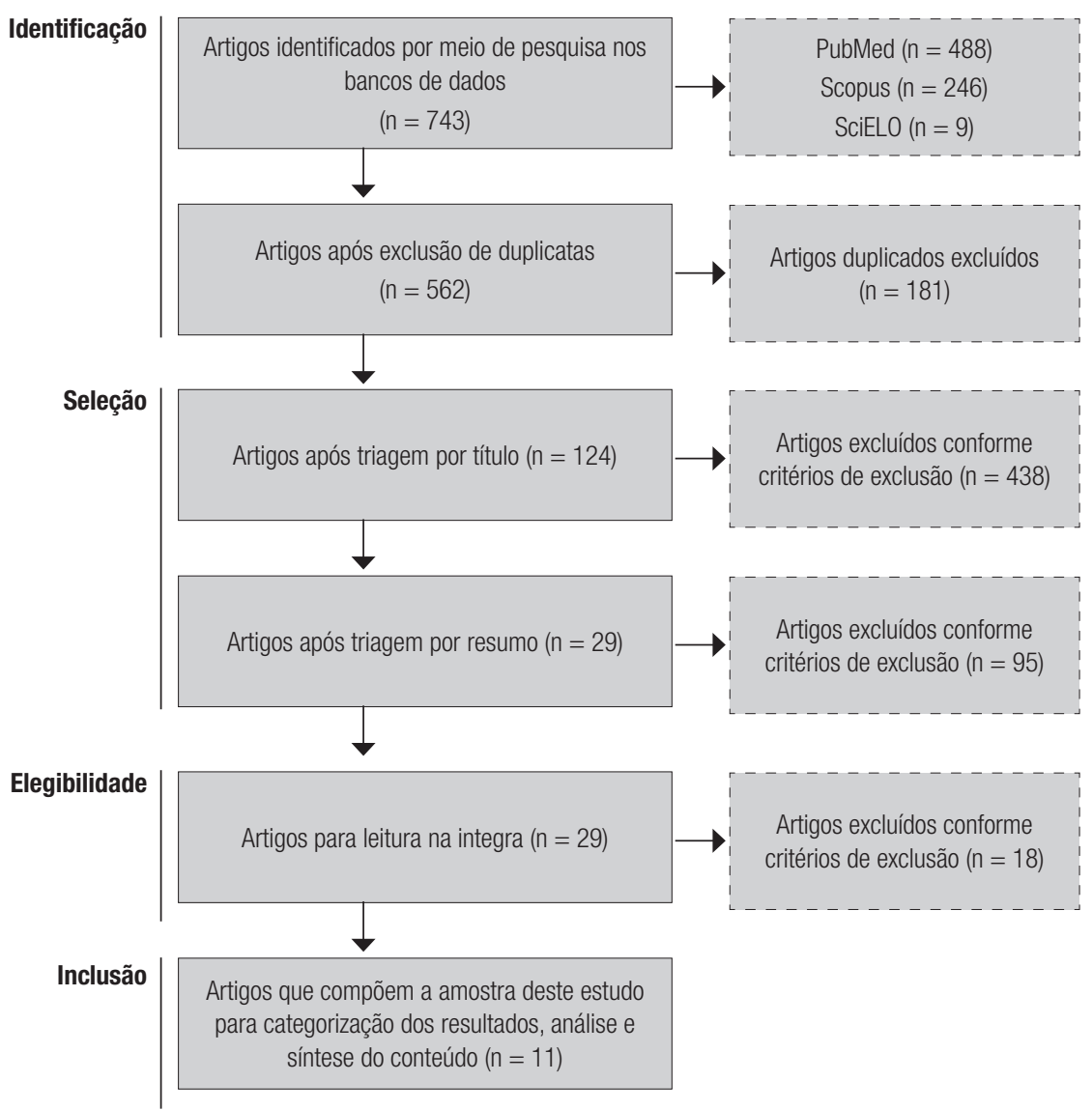

Figura 1. Fluxograma da seleção dos artigos.

Tabela 1. Artigos que tratam dos aspectos subjetivos na adesão ao tratamento do transtorno bipolar

\begin{tabular}{|c|c|c|c|}
\hline $\begin{array}{l}\text { Autores, ano de } \\
\text { publicação e } \\
\text { país }\end{array}$ & Tipo de estudo e amostra & Título em português & Resultados \\
\hline $\begin{array}{l}\text { Averous et al. } \\
\text { (2018) - França }\end{array}$ & $\begin{array}{l}\text { Estudo transversal com } 38 \\
\text { sujeitos }\end{array}$ & $\begin{array}{l}\text { Percepções de doença e adesão ao transtorno } \\
\text { bipolar: um estudo exploratório }\end{array}$ & $\begin{array}{l}\text { Pacientes com menos sentimentos negativos em relação ao } \\
\text { TB, percepção de maior controle do tratamento e que } \\
\text { percebem menos consequências em virtude dele tiveram } \\
\text { maior adesão ao tratamento. }\end{array}$ \\
\hline $\begin{array}{l}\text { Arvilommi et al. } \\
\text { (2014) - Finlândia }\end{array}$ & $\begin{array}{l}\text { Estudo longitudinal } \\
\text { prospectivo com } 168 \text { sujeitos }\end{array}$ & $\begin{array}{l}\text { Preditores da adesão ao tratamento } \\
\text { psicofarmacológico e psicossocial em transtornos } \\
\text { bipolares I ou II - estudo prospectivo de } 18 \text { meses }\end{array}$ & $\begin{array}{l}\text { Os motivos para a não adesão foram efeitos colaterais, falta } \\
\text { de motivação e atitudes negativas em relação ao } \\
\text { tratamento. }\end{array}$ \\
\hline $\begin{array}{l}\text { Bowskill et al. } \\
\text { (2007) - Inglaterra }\end{array}$ & $\begin{array}{l}\text { Estudo transversal com } 223 \\
\text { sujeitos }\end{array}$ & $\begin{array}{l}\text { Percepções dos pacientes sobre as informações } \\
\text { recebidas sobre medicamentos prescritos para } 0 \\
\text { transtorno bipolar: implicações para a escolha informada }\end{array}$ & $\begin{array}{l}\text { Baixa adesão relacionada à insatisfação com as } \\
\text { informações recebidas sobre os medicamentos. }\end{array}$ \\
\hline $\begin{array}{l}\text { Navarro et al. } \\
(2016) \text { - Espanha }\end{array}$ & $\begin{array}{l}\text { Estudo transversal com } 76 \\
\text { sujeitos }\end{array}$ & $\begin{array}{l}\text { Variáveis associadas à não adesão em pacientes } \\
\text { clinicamente estáveis com transtorno bipolar }\end{array}$ & $\begin{array}{l}\text { Pacientes não aderentes tiveram maior preocupação com } \\
\text { relação aos medicamentos, além de pior funcionamento } \\
\text { global, maior número de episódios, mais morbidades } \\
\text { psiquiátricas e uso prejudicial de drogas. }\end{array}$ \\
\hline $\begin{array}{l}\text { Sajatovic et al. } \\
\text { (2005) - EUA }\end{array}$ & $\begin{array}{l}\text { Estudo longitudinal } \\
\text { prospectivo com } 52 \text { sujeitos }\end{array}$ & $\begin{array}{l}\text { Atitudes em relação ao modelo de prática } \\
\text { colaborativa e adesão ao tratamento entre indivíduos } \\
\text { com transtorno bipolar }\end{array}$ & $\begin{array}{l}\text { Alianças terapêuticas colaborativas favorecem maior } \\
\text { adesão. }\end{array}$ \\
\hline $\begin{array}{l}\text { Sajatovic et al. } \\
\text { (2011) - EUA }\end{array}$ & $\begin{array}{l}\text { Estudo transversal com } 20 \\
\text { sujeitos }\end{array}$ & $\begin{array}{l}\text { Experiência de doença e razões para a não adesão } \\
\text { entre indivíduos com transtorno bipolar que são } \\
\text { pouco aderentes à medicação }\end{array}$ & $\begin{array}{l}\text { Os motivos para não tomar a medicação foram: } \\
\text { esquecimento, efeitos colaterais, ambientes familiares } \\
\text { desorganizados, medo de tomar medicamentos a longo } \\
\text { prazo e informações insuficientes sobre o transtorno. }\end{array}$ \\
\hline $\begin{array}{l}\text { Strauss \& Johnson } \\
\text { (2006) - EUA }\end{array}$ & $\begin{array}{l}\text { Estudo longitudinal } \\
\text { prospectivo com } 58 \text { sujeitos }\end{array}$ & $\begin{array}{l}\text { Papel da aliança de tratamento no manejo clínico do } \\
\text { transtorno bipolar: alianças mais fortes predizem } \\
\text { prospectivamente menos sintomas maníacos }\end{array}$ & $\begin{array}{l}\text { Alianças terapêuticas fortes estavam relacionadas a menos } \\
\text { atitudes negativas sobre os medicamentos, menos } \\
\text { sentimento de estigma e maior suporte social. }\end{array}$ \\
\hline
\end{tabular}




\begin{tabular}{|c|c|c|c|}
\hline $\begin{array}{l}\text { Autores, ano de } \\
\text { publicação e } \\
\text { país }\end{array}$ & Tipo de estudo e amostra & Título em português & Resultados \\
\hline $\begin{array}{l}\text { Clatworthy et al. } \\
\text { (2009) - Inglaterra }\end{array}$ & $\begin{array}{l}\text { Estudo transversal com } 223 \\
\text { sujeitos }\end{array}$ & $\begin{array}{l}\text { Compreender a não adesão à medicação em } \\
\text { transtornos bipolares usando uma Estrutura de } \\
\text { Preocupações de Necessidade }\end{array}$ & $\begin{array}{l}\text { Má adesão ao tratamento foi relatado por } 30 \% \text { dos sujeitos } \\
\text { e foi atribuída a dúvidas com relação a necessidade de } \\
\text { estar em tratamento e preocupações atreladas a ele. }\end{array}$ \\
\hline $\begin{array}{l}\text { Inder et al. (2010) } \\
\text { - Nova Zelândia }\end{array}$ & $\begin{array}{l}\text { Estudo longitudinal } \\
\text { prospectivo com } 15 \text { sujeitos }\end{array}$ & $\begin{array}{l}\text { "Eu realmente não sei se ainda está lá”: Aceitação } \\
\text { Ambivalente de um Diagnóstico de Transtorno Bipolar }\end{array}$ & $\begin{array}{l}\text { Experiências negativas com relação ao tratamento } \\
\text { contribuíram para sentimentos ambivalentes sobre } 0 \\
\text { diagnóstico, recaídas e má adesão. }\end{array}$ \\
\hline $\begin{array}{l}\text { Cruz et al. (2011) } \\
\text { - Brasil }\end{array}$ & $\begin{array}{l}\text { Estudo transversal com } 17 \\
\text { sujeitos }\end{array}$ & $\begin{array}{l}\text { Terapêutica medicamentosa: adesão, conhecimento e } \\
\text { dificuldades de idosos com transtorno bipolar }\end{array}$ & $\begin{array}{l}\text { Apontou má adesão relacionada a déficits no conhecimento } \\
\text { sobre os fármacos, tendo como causas: desejo de encerrar } \\
\text { o tratamento, efeitos colaterais, dúvidas sobre a } \\
\text { necessidade do tratamento e outros. }\end{array}$ \\
\hline $\begin{array}{l}\text { Miasso et al. } \\
\text { (2009) - Brasil }\end{array}$ & $\begin{array}{l}\text { Estudo transversal com } 21 \\
\text { sujeitos }\end{array}$ & $\begin{array}{l}\text { Transtorno afetivo bipolar: adesão ao medicamento e } \\
\text { satisfação com o tratamento e orientações da equipe } \\
\text { de saúde de um Núcleo de Saúde Mental }\end{array}$ & $\begin{array}{l}\text { Apenas } 28,5 \% \text { eram aderentes, } 57,2 \% \text { dos sujeitos são } \\
\text { não aderentes por comportamentos não intencionais e } \\
14,3 \% \text {, por comportamentos intencionais. }\end{array}$ \\
\hline
\end{tabular}

Foram analisados 11 artigos, 9 em língua inglesa e apenas dois em português. Onze estudos são observacionais; desses, quatro são longitudinais prospectivos e sete, transversais. Em sua maioria, foram utilizadas abordagens mistas, mensurações tanto quantitativas como qualitativas. O estudo mais recente foi publicado em 2018 e o mais antigo, em 2005. Conforme esquematizado na figura 2, a adesão ao tratamento foi associada às atitudes resultantes das percepções do sujeito e às atitudes por influência de pessoas próximas.

\section{Adesão ao tratamento como resultado da percepção do sujeito sobre o transtorno e o tratamento}

No conjunto dos nove artigos ${ }^{16-24}$ que abordam as atitudes como resultado da percepção dos sujeitos, há percepções relacionadas ao transtorno e relativas ao tratamento. Esse conjunto de percepções está, por sua vez, relacionado à resposta emocional expressa, compondo o universo de vivências do sujeito. Nas percepções do transtorno, são incluídos: nível de compreensão sobre o TB e aceitação ou negação da condição. Entre os fatores que compõem a percepção sobre o tratamento, são colocados: temor em relação aos efeitos colaterais; dúvidas sobre a eficácia e a utilidade do tratamento; preocupações com as medicações, como temer usá-las pelo resto da vida ou querer ajustá-las; percepção de que os fármacos trazem consequências ou benefícios e comportamentos negativos intencionais ou não intencionais, tais como perda e esquecimento dos medicamentos, fruto de um gerenciamento inadequado dos fármacos.

O estudo mais abrangente nesse segmento das atitudes provenientes das percepções dos sujeitos, tanto com relação ao tamanho da amostra como também por fazer uma avaliação prospectiva, foi conduzido por Arvilommi et al.." Os autores avaliaram 168 pacientes de três cidades finlandesas diagnosticados com TB dos tipos I e I| que estavam internados ou em seguimento ambulatorial ao longo de 18 meses, com intuito de investigar se os pacientes, ao longo do tempo do estudo, continuaram com o tratamento prescrito e, caso contrário, quais os motivos para a descontinuação. Observaram que 1/4 dos sujeitos interrompeu o uso dos psicofármacos e um terço dos que mantiveram o uso da medicação fez uso dela de forma irregular. Entre as principais razões, estão: efeitos colaterais, pouca motivação e atitudes negativas com o tratamento. Também são frequentes questões relativas ao não uso por perder ou se esquecer de tomar a medicação, achar o tratamento ineficaz, ter problemas com álcool, problemas físicos e incompreensão e querer ajustar a dose.

Outros estudos chegaram a conclusões similares, identificando que pacientes não aderentes apresentam pior compreensão do transtorno e maior temor com relação aos medicamentos ${ }^{16,18-20}$. Geralmente, a preocupação com as medicações pode surgir tanto pelo fato de o paciente ter experimentado efeitos colaterais reais, mas também pela possibilidade de um dia vir a vivenciá-los. ${ }^{24}$. Mas quando os pacientes têm a percepção de que o tratamento de fato consegue controlar os sintomas e enxergam menos consequências advindas do tratamento, eles tendem a ter melhor adesão ${ }^{16}$.

O estudo quali-quantitativo de Miasso et al. ${ }^{24}$ entrevistou 21 sujeitos com TB e verificou que 58,2\% deles não aderem ao tratamento por comportamentos não intencionais e 14,3\%, por comportamentos intencionais. É interessante pontuar que, mesmo entre os pacientes aderentes à medicação, metade deles tem dúvidas sobre a eficácia dos medicamentos (16\%) ou está insatisfeita com eles (33\%). Algo semelhante também foi observado nos não aderentes não intencionais: metade deles refere insatisfação com os medicamentos. Embora a grande maioria dos sujeitos esteja satisfeita com as informações prestadas pela equipe de saúde, os pesquisadores constataram que muitos assumem uma postura passiva no tratamento e apresentam sentimentos e opiniões ambivalentes a respeito dos medicamentos. Os pesquisadores ainda apontam que é de extrema importância que os profissionais de saúde estejam abertos para escutar os pacientes e 


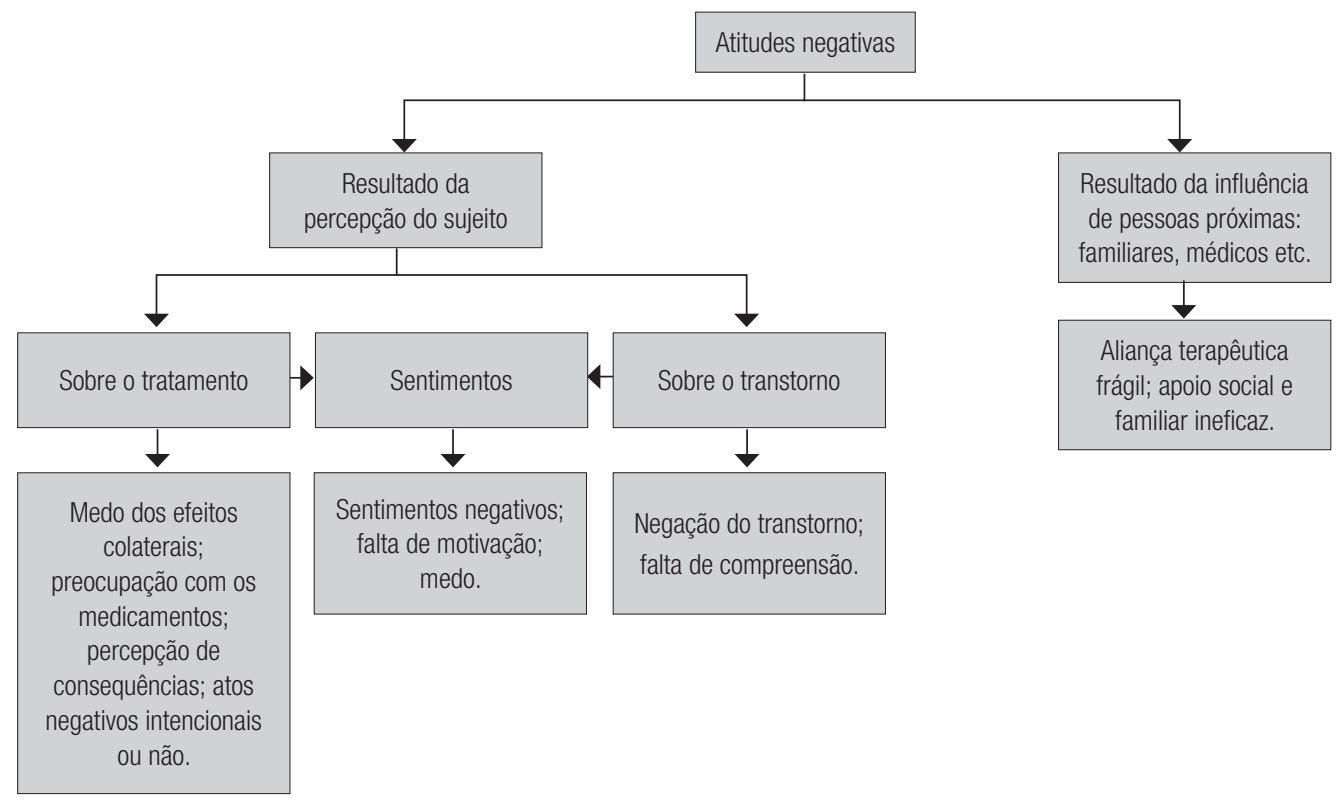

Figura 2. Aspectos subjetivos relacionados a má adesão ao tratamento do transtorno bipolar.

acolher suas angústias e que o tratamento seja pautado em uma metodologia colaborativa e de corresponsabilização por parte dos usuários.

\section{Adesão ao tratamento como resultado da influência de pessoas próximas}

Com relação às atitudes provenientes da influência de pessoas próximas, destacam-se dois estudos que abordam a importância de uma boa aliança terapêutica ${ }^{25,26}$ e o estudo de Sajatovic et al..20, que foca no apoio social. A influência dessas duas esferas pode resultar em comportamentos de engajamento ou de desinvestimento no tratamento, a depender da qualidade da relação médico-paciente, do grau de conhecimento e da participação da família no tratamento.

Nesse sentido, o estudo de Sajatovic et al. ${ }^{25}$ encontrou bons índices de adesão ao tratamento ao avaliar a implementação de um programa terapêutico que tinha como foco melhorar a capacidade dos pacientes de participar do tratamento de forma colaborativa. Entre os principais elementos constituintes desse programa, estavam: definições colaborativas de problemas; construção conjunta de metas e planejamento; oferta de um continuum de serviços de autogerenciamento e suporte; e acompanhamento ativo. Recomendações específicas aos psiquiatras e equipes de saúde incluem: considerar a opinião do paciente; considerar tratamentos alternativos; ser um bom ouvinte e ter sensibilidade com relação aos sentimentos dos pacientes. Assim, uma boa aliança terapêutica pode minimizar parte das atitudes negativas dos pacientes, pois pacientes que apresentam relação satisfatória com a equipe de saúde tendem a procurá-la em busca de orientações sobre o tratamento, além de representar um elemento de apoio e escuta em um contexto social que na maioria das vezes os rejeita e estigmatiza ${ }^{26}$.

Por fim, sobre a influência que o meio social pode ter nas atitudes em relação ao tratamento, o estudo de Sajatovic et al. alerta que muitos pacientes não aderem ao tratamento porque pessoas próximas, muitas vezes da própria família, os influenciam a não tomar as medicações. Assim, é de fundamental importância que as equipes de saúde estejam atentas para que, além dos pacientes, os familiares sejam incluídos na esfera de cuidados da equipe e sejam bem instruídos com relação ao tratamento e ao transtorno ${ }^{20}$.

\section{DISCUSSÃO}

Fazendo um paralelo dos achados desta pesquisa com os dados da literatura, outros estudos também apontam a relação entre má adesão e atitudes negativas quanto ao uso dos psicofármacos, especialmente com relação aos efeitos colaterais $s^{21,27,28}$. Horne et al. ${ }^{29}$ ainda apontam que pacientes com doenças crônicas tendem a estabelecer uma relação com os medicamentos permeada pela avaliação do custo-benefício, pesando suas preocupações versus necessidades, o que pode resultar em comportamentos mais ou menos aderentes. Fisher et al. ${ }^{30}$ apontam que os pacientes tendem a se engajar mais quando o tratamento está ancorado em uma abordagem centrada no paciente, em vez das abordagens tradicionais pautadas em práticas paternalistas. E a colaboração e o sentimento de participação na tomada de decisões são a principal dimensão de uma boa aliança terapêutica, segundo Sylvia et al. ${ }^{31}$. 
A colaboração deve ser a base para o relacionamento da equipe com o paciente e, em especial, a relação médico-paciente. Estudos mostram que os médicos tendem a subestimar a carga emocional do transtorno, que tal carga tem impacto na adesão e que os motivos relatados por médicos e pacientes para a má adesão divergem, o que é reflexo de falhas na comunicação e confiança ${ }^{32}$. Assim, estratégias que tenham empatia e considerem a perspectiva dos pacientes podem facilitar a discussão com os médicos sobre os motivos que levam os pacientes a não aderirem aos medicamentos ${ }^{33}$.

Vem ganhando força nas últimas décadas o entendimento de que a psicoterapia e o suporte social são mecanismos essenciais no tratamento, amparados na visão de modelos multifatoriais para a compreensão do processo saúde-doença. Com relação às estratégias psicossociais, os autores apontam para a sua eficácia na melhor adesão ao tratamento no TB ${ }^{34,35}$, uma vez que esses modelos são centrados no sujeito e sua experiência subjetiva, além de a colaboração e a construção conjunta serem a base desse tipo de intervenção. A terapia cognitivo-comportamental, as estratégias psicoeducativas e as terapias familiares nas modalidades individual ou em grupo são elencadas como as que obtiveram melhores resultados segundo estudos com grupo controle. Em geral, as intervenções psicossociais buscam: elaborar os significados e crenças dos pacientes e/ou familiares em relação ao tratamento; resolver problemas; empoderar o paciente no processo de cuidado; ajudar os pacientes a identificarem e controlarem os sintomas; desenvolver estratégias de enfrentamento em relação a situações estressantes; estimular o suporte familiar e reduzir traumas e estigmas relacionados ao TB. Algumas abordagens focarão mais em alguns desses objetivos e outros podem ser acrescentados ${ }^{36}$. Em conjunto com o uso dos medicamentos, o emprego de intervenções psicossociais aumenta a adesão terapêutica e melhora a qualidade de vida e a readaptação social ${ }^{37}$.

No que concerne ao suporte social, é necessário antes compreender que ele pode ser dividido em estrutural e funcional. O suporte social estrutural diz respeito à rede familiar e social disponível que pode oferecer ajuda e cuidado, enquanto o suporte social funcional está relacionado à dimensão qualitativa, ao modo como recursos emocionais, materiais e afetivos são empregados. Essa consideração é salutar, uma vez que é comum que as avaliações de suporte social nas instituições tendem a se restringir apenas a presença de cônjuge, composição familiar, existência de algum confidente e engajamento em atividades sociais, esquecendo-se da esfera relacional de como esses atores envolvidos ofertam o cuidado. Embora sejam poucos os estudos a respeito dessa dimensão no TB, eles apontam que o suporte social tem papel importante para amenizar os impactos negativos associados ao transtorno. Também atua na redução de sintomas a longo prazo, favorecendo posturas mais assertivas no tratamento e menos estigmas ${ }^{38}$.

\section{CONCLUSÕES}

No campo dos aspectos subjetivos, várias atitudes contribuem para o não seguimento do tratamento. Podemos dividir tais aspectos em dois campos, como sendo: resultado da percepção dos pacientes sobre o transtorno e o tratamento, e resultado da influência de pessoas próximas. No que se refere ao resultado da percepção dos pacientes sobre o transtorno e o tratamento, destacam-se os fatores: medo dos efeitos colaterais; preocupação com os medicamentos; percepção de consequências; atos negativos intencionais ou não; negação do transtorno; falta de compreensão; sentimentos negativos e falta de motivação. Como resultado da influência de pessoas próximas, temos os fatores: alianças terapêuticas frágeis e apoio social ineficaz. Compreender essas variáveis é de suma importância, pois as vivências, crenças e percepções dos pacientes podem ser fatores para engajamento ou descrédito quanto à eficácia e à necessidade de tratamento. Assim sendo, a satisfação do paciente tem forte influência na adesão.

Contudo, considerar as vivências das pessoas com TB não é apenas um meio para melhorar a adesão terapêutica, mas é principalmente uma forma de garantir um tratamento que respeite a dignidade desses sujeitos, que são esposas, filhos, irmãos ou mães de milhares de pessoas.

\section{CONTRIBUIÇÕES INDIVIDUAIS}

Kaliano Márcio de Queiroz Costa - Participou da elaboração do desenho do estudo, da análise e interpretação dos resultados e da redação do artigo e aprovou o texto final.

Rachel Medeiros de Góes - Participou da elaboração do desenho do estudo, da análise e interpretação dos resultados, da correção do artigo e da revisão crítica do conteúdo apresentado e aprovou o texto final.

Maria Mabel Nunes de Morais - Participou da análise e interpretação dos resultados, da correção do artigo e da revisão crítica do conteúdo e aprovou o texto final.

\section{CONFLITOS DE INTERESSES}

Os autores deste estudo - Kaliano Marcio de Queiroz Costa, Maria Mabel Nunes de Morais e Rachel Medeiros de Góes - não receberam qualquer incentivo financeiro ou material de entidades ou organizações em razão da temática objeto de estudo desta pesquisa e que pudesse configurar conflito de interesse.

\section{AGRADECIMENTOS}

Agradeço à Dra. Neucine Gomes da Silva, professora do Departamento de Psicologia da Universidade Federal 
do Rio Grande do Norte, e à Dra. Cristiane Santos Sânzio Gurgel, servidora da Secretaria de Saúde do Estado do Rio Grande do Norte, por participarem como avaliadoras deste estudo que consistiu em trabalho de conclusão de curso para a obtenção de título de especialista no Programa de Residência Integrada Multiprofissional em Saúde da Universidade Federal do Rio Grande do Norte (UFRN). Agradecimento especial também a minha mãe, diagnosticada e em tratamento para TB desde que nasci - conviver com o transtorno na condição de filho despertou o meu interesse por uma assistência de qualidade para as pessoas com essa condição.

\section{REFERÊNCIAS}

1. Merikangas KR, Jin R, He JP, Kessler RC, Lee S, Sampson NA, et al. Prevalence and correlates of bipolar spectrum disorder in the world mental health survey initiative. Arch Gen Psychiatry. 2011;68:241-51.

2. Goldberg JF, Harrow M, Grossman LS. Recurrent Affective Syndromes in Bipolar and Unipolar Mood Disorders at Follow-Up. Br J Psychiatry. 1995;166(3):382-5.

3. Magalhães PVS, Costa MH, Pinheiro RT. Epidemiologia do transtorno bipolar. In: Kapczinski F, Quevedo J (Org.). Transtorno bipolar - teoria e clínica. 2a ed. Porto Alegre: Artmed; 2016. p. 21-31.

4. Gama CS, André ASG, Filtman A, Rosa AR. Tratamento farmacológico do transtorno bipolar. In: Kapczinski F, Quevedo J (Org.). Transtorno bipolar - teoria e clínica. 2a ed. Porto Alegre: Artmed; 2016. p. 197-214.

5. American Psychiatry Association (APA). Practice Guideline for the Treatment of Patients with Bipolar Disorder. 2th ed. Washington: APA; 2010.

6. Johnson SL, Fristad M. Bipolar disorders across the lifespan. J Clin Psychol. 2008;64:365-7.

7. Neves FS, Lima IMM, Malloy-Diniz LF. Tratamento psicoterápico do transtorno bipolar. In: Kapczinski F, Quevedo J (Org.). Transtorno bipolar - teoria e clínica. 2a ed. Porto Alegre: Artmed; 2016. p. 231-42.

8. Cardoso L, Galera SAF. Doentes mentais e seu perfil de adesão ao tratamento psicofarmacológico. Rev Esc Enferm USP. 2009;43(1):161-7.

9. Lingam R, Scott J. Treatment non-adherence in affective disorders. Acta Psychiatr Scand. 2002:105:164-72.

10. Castro-Costa E, Silva AG. A adesão terapêutica no transtorno bipolar. Rev Debates Psiquiatr. 2011;5:8-12.

11. Anna KM, Cardoso BM, Bücker J. Comorbidades psiquíatricas no transtorno bipolar. In: Kapczinski F, Quevedo J (Org.). Transtorno bipolar - teoria e clínica. 2a ed. Porto Alegre: Artmed; 2016. p. 153-64

12. Kessler RC, Merikangas KR, Wang PS. Prevalence, comorbidity, and service utilization for mood disorders in the United States at the beginning of the twenty-first century. Annu Rev Clin Psychol. 2007;3:137-58.

13. Galvão TF, Pansani TSA, Harrad DP. Principais itens para relatar revisões sistemáticas e meta-análises: a recomendação PRISMA. Epidemiol Serv Saúde. 2015;24(2):335-42.

14. Souto RQ, Lima KSA, Pluye P, Hong QN, Barbosa KE, Araújo GKN. Tradução e adaptação transcultural do instrumento Mixed Methods Appraisal Tool ao contexto brasileiro. Rev Fun Care Online. 2020;12:510-6.

15. Sandelowski M, Barroso J. Handbook for Synthesizing Qualitative Research. 1st ed. New York: Springer Publishing Company; 2007. p. 312.

16. Averous P, Charbonnier E, Lagouanelle-Simeoni MC, Prosperi A, Dany L. Illness perceptions and adherence in bipolar disorder: An exploratory study. Compr Psychiatry. 2018:80:109-15.
17. Arvilommi P, Suominen K, Mantere O, Leppamaki S, Valtonen H, Isometsa E. Predictors of adherence to psychopharmacological and psychosocial treatment in bipolar I or II disorders - an 18-month prospective study. J Affect Disord. 2014;155:110-7.

18. Bowskill R, Clatworthy J, Parham R, Rank T, Horne R. Patients' perceptions of information received about medication prescribed for bipolar disorder: implications for informed choice. J Affect Disord. 2007;100(1-3):253-7.

19. Navarro S, Rodríguez F, Acosta FJ, García-Bello M. Variables associated with nonadherence in clinically stable patients with bipolar disorder. Actas Esp Psiquiatr. 2016;44:157.

20. Sajatovic M, Levin J, Fuentes-Casiano E, Cassidy K, Tatsuoka C, Jenkins J. Illness experience and reasons for nonadherence among individuals with bipolar disorder who are poorly adherent with medication. Compr Psychiatry. 2011;52(3):280-7.

21. Clatworthy J, Bowskill R, Parham R, Rank T, Scott J, Horne R. Understanding medication non-adherence in bipolar disorders using a Necessity-Concerns Framework. J Affect Disord. 2009;116 (1-2):51-5.

22. Inder ML, Crowe MT, Joyce PR, Moor S, Carter JD, Luty SE. "I Really Don't Know Whether it is Still There": Ambivalent Acceptance of a Diagnosis of Bipolar Disorder. Psychiatr Quarterly. 2010;81:157-65.

23. Cruz LP, Miranda PM, Vedana KGG, Miasso Al. Terapêutica medicamentosa: adesão, conhecimento e dificuldades de idosos com transtorno bipolar. Rev Latino-Am Enfermagem. 2011;19(4):944-52.

24. Miasso Al, Monteschi M, Giacchero KG. Transtorno afetivo bipolar: adesão do medicamento e satisfação com 0 tratamento e orientações da equipe de saúde de um núcleo de saúde mental. Rev Latino-Am Enfermagem. 2009;17(4):548-56.

25. Sajatovic M, Davies M, Bauer MS, McBride L, Hays RW, Safavi R. Attitudes regarding the collaborative practice model and treatment adherence among individuals with bipolar disorder. Compr Psychiatry. 2005;46:272-7.

26. Strauss JL, Johnson SL. Role of treatment alliance in the clinical management of bipolar disorder: stronger alliances prospectively predict fewer manic symptoms. Psychiatry Res. 2006; 145(2-3):215-23

27. Leclerc $E$, Mansur RB, Brietzke E. Determinants of adherence to treatment in bipolar disorder: A comprehensive review. J Affect Disord. 2013;149:247-52.

28. Busby KK, Sajatovic M. Review: patient, treatment, and systems-level factors in bipolar disorder nonadherence: a summary of the literature. CNS Neurosci Ther. 2010;16:308-15.

29. Horne R, Weinman J, Hankins M. The Beliefs about Medicines Questionnaire: The development and evaluation of a new method for assessing the cognitive representation of medication. Psychol Health. 1999;14(1):1-24.

30. Fisher A, Manicavasagar V, Sharpe L, Laidsaar-Powell R, Juraskova I. Identifying and Addressing Barriers to Treatment Decision-making in Bipolar II Disorder: Clinicians' Perspective. Australian Psychologist. 2017;53(1):40-51.

31. Sylvia LG, Hay A, Ostacher MJ, Miklowitz DJ, Nierenberg AA, Thase ME, et al. Association between therapeutic alliance, care satisfaction, and pharmacological adherence in bipolar disorder. J Clin Psychopharmacol. 2013;33:343-50

32. Pope M, Scott J. Do clinicians understand why individuals stop taking lithium? J Affect Disord. 2003;74:287-91.

33. Heszen-Klemens I, Lapinska E. Doctor-patient interaction, patients' health behavior and effects of treatment. Soc Sci Med. 1984;19(1):9-18.

34. Scott J, Colom F, Popova E, Benabarre A, Cruz N, Valenti M, et al. Long-term mental health resource utilization and cost of care following group psychoeducation or unstructured group support for bipolar disorders: a cost-benefit analysis. J Clin Psychiatry. 2009;70(3):378-86.

35. Miklowitz DJ, Scott J. Psychosocial treatments for bipolar disorder: cost-effectiveness, mediating mechanisms, and future directions. Bipolar Disord. 2009;11(2):110-22.

36. Bio D, Gomes BC. Abordagens Psicossociais. Rev Debates Psiquiatr. 2011;5:38-48.

37. Souza FGME, Reboucas DB, Luna JRG, Matos KJN, Barros EM, Vasconcelos NC, et al. Tratamento de manutenção no transtorno bipolar. Rev Debates Psiquiatr. 2011;5:24-33.

38. Studart PM, Bezerra Filho S, Studart ABD, Almeida AG, Miranda-Scippa A. Social support and bipolar disorder. Arch Clin Psychiatry. 2015;42(4):95-9. 